\title{
Long-Term Expansion and Sensitization of Mechanosensory Receptive Fields in Aplysia Support an Activity-Dependent Model of Whole-Cell Sensory Plasticity
}

\author{
Allen J. Billy and Edgar T. Walters \\ Department of Physiology and Cell Biology, University of Texas Medical School at Houston, Houston, Texas 77225
}

Long-term changes in peripheral receptive field properties of mechanosensory/nociceptive neurons were investigated 1-3 weeks after noxious stimulation. Noxious stimuli consisted of a deep penetrating cut through the middle of the tail, strong electric shock applied to the tail surface, or a combination of deep and superficial tail stimulation. Action potentials evoked in the tail were monitored with intracellular electrodes in central somata of tail sensory neurons. Three long-term changes in receptive field properties were produced in the region of noxious stimulation: (1) mechanosensory thresholds decreased, (2) receptive field areas increased, and (3) the percentage of cells showing receptive field extension across the tail midline increased. Sizes and shapes of individual receptive fields did not vary during extensive testing of tails perfused with artificial seawater or during testing in cobalt solutions that block synaptic transmission. This stability of receptive field geometry, coupled with the observation that increased peripheral excitability in these cells does not increase receptive field size, suggests that long-term receptive field alterations involve growth of peripheral sensory processes. A model is proposed in which the signaling strength of the entire sensory cell increases in response to trauma of its receptive field. In this model longterm enhancement of central and peripheral sensory responsiveness is selectively triggered by activity dependent extrinsic modulation of the centrally located soma, which accelerates synthesis of growth-associated proteins used in collateral and regenerative sprouting of traumatized peripheral processes.

Strong electric shock (Pinsker et al., 1973; Frost et al., 1985; Walters, 1987a) or traumatic mechanical stimuli (Walters, 1987a) delivered to intact Aplysia can produce behavioral sensitization lasting weeks. These stimuli are considered noxious because they cause overt tissue injury and/or trigger afferent activity normally produced by body wall injury (Walters et al., 1983). Noxious stimuli can enhance central signaling properties of mechanosensory neurons for at least $1 \mathrm{~d}$ following stimulation by facil-

Received May 31, 1988; revised Aug. 11, 1988; accepted Sept. 12, 1988.

We thank Dr. A. Eskin for comments on the manuscript, and J. Pastore and D. Morse for illustrations. This work was supported by National Institutes of Mental Health Grant MH38726, National Institutes of Health Research Career Development Award NS00848, and the Chicago Community Trust/Searle Scholars Program.

Correspondence should be addressed to Dr. Edgar T. Walters, Department of Physiology and Cell Biology, University of Texas Medical School at Houston P.O. Box 20708, Houston, TX 77225.

Copyright (C) 1989 Society for Neuroscience $0270-6474 / 89 / 041254-09 \$ 02.00 / 0$ itating synaptic transmission to central follower cells (cf. Bailey and Chen, 1983; Frost et al., 1985; Walters, 1987b) and perhaps by increasing central excitability (Scholz and Byrne, 1987; Walters, 1987b). Long-lasting increases in central synaptic transmission and soma excitability also occur when nerve stimulation (Hawkins et al., 1983; Walters and Byrne, 1985) or 5-HT application (Montarolo et al., 1986; Dale et al., 1987, 1988) is used as a substitute for skin stimulation in reduced preparations. In contrast, little is known about the capacity of the peripheral regions of mechanosensory neurons for persistent modification. Clark and Kandel $(1984,1987)$ reported that 5-HT application enhances transmission from peripheral synapses of siphon mechanosensory neurons, and indirect evidence (Klein et al., 1986; Walters, 1987a, b; Billy and Walters, 1988) suggests that sensory transduction or spike initiation in the periphery may be facilitated shortly after noxious stimulation.

The many changes implicated in mechanosensory neurons following noxious stimulation raise interesting functional questions. Does long-term plasticity affect the signaling effectiveness of the whole cell, including its peripheral receptive components? What is the biological significance of activity-dependent enhancement of cellular plasticity in mechanosensory neurons (I Iawkins et al., 1983; Walters and Byrne, 1983, 1985)? Why are activity-dependent effects prominent in the cell soma (Hawkins et al., 1983; Ocorr et al., 1985; Walters and Byrne, 1985; Walters, 1987b) - a site removed from the direct signaling pathway between the receptive field and the sensory neuron's synaptic connections to the CNS? The experiments reported here begin to address these questions by considering nociceptive functions of Aplysia mechanosensory neurons (Walters et al., 1983; Walters, 1987b). A nociceptive role suggested that some of the alterations being studied in sensory neurons contribute to the functional equivalent of primary "hyperalgesia" in this animal (Walters, 1987a). Hyperalgesia has been associated with peripheral sensitization of nociceptors in mammals (e.g., Lewis, 1942; Fitzgerald, 1979; Coderre and Melzack, 1987), raising the possibility that central facilitation in Aplysia sensory neurons is coordinated with sensitization of their peripheral processes. Since injury-induced hyperalgesia can last for months or longer (e.g., Woolf, 1984) and regenerating nociceptors can show dramatic reductions in mechanosensory threshold (Dickhaus et al., 1976), it was of interest to see if injury in Aplysia would cause longterm reductions in mechanosensory threshold and changes in receptive field geometry. The present study indicates that both long-term sensitization and expansion of mechanosensory receptive fields occur near a site of injury in Aplysia. Furthermore, these results and others are consistent with a growth-related 
function of trauma-induced, activity-dependent modulation of the sensory neuron soma. Some of these results have been described in abstract form (Billy and Walters, 1987).

\section{Materials and Methods}

Aplysia californica (100-350 gm), supplied by Alacrity Marine Biological Services (Redondo Beach, CA) and Sea Life Supply (Sand City, CA), were maintained in artificial seawater ("Instant Ocean," Carolina Biological Supply) at $17-19^{\circ} \mathrm{C}$ on a $12 \mathrm{hr}$ light-dark cycle. Animals were fed sufficient lettuce at $\mathbf{2}-\mathbf{3}$ d intervals to maintain a relatively constant body weight.

Noxious stimulation procedures. Noxious stimuli were delivered to 3 experimental groups; a fourth group was used as an unstimulated control. Animals in the Cut Alone group $(n=26)$ received a deep, transverse razor cut from the lateral margin of the middle of the tail to approximately the midline. In all cases the cut completely penetrated both the dorsal and ventral body wall (see Fig. 4 for examples). No anesthesia was used. During the period of the study (1-3 weeks) opposite edges of the cut did not fuse back together. Animals in the Superficial Stimulation group $(n=18)$ received 20 trains $(0.5 \mathrm{sec}$ each) of electric shock (10$40 \mathrm{~mA}, 60 \mathrm{~Hz} \mathrm{ac}$ ) at $5 \mathrm{sec}$ intervals through hand-held capillary electrodes pressed firmly against the center of the dorsal tail surface (Walters, 1987a). Shock often caused fading of cutaneous pigmentation but did not produce lesions that penetrated the skin. Animals in the Cut + Superficial Stimulation group $(n=31)$ received the deep cut followed immediately by either electric shock $(n=16)$ or mechanical stimulation $(n=15)$. The latter consisted of a 2 min series of 20 pinches (about 0.5 sec each) delivered to the dorsal surface of the tail with toothed forceps having tips 0.75 or $1.5 \mathrm{~mm}$ thick. Animals in the Control group $(n=$ 16) received no stimulation except normal handling. The tester was nominally "blind" to the particular group to which a preparation belonged. The blind was limited, however, as even superficial noxious stimuli often left discolorations that could be seen when the tail surface was pulled taut and flattened. Discolorations were not noticed in previous studies using the free tail (Walters and Erickson, 1986; Walters, 1987a) or loosely pinned tail preparation (Walters, 1987b) because they had been obscured by folds in the body wall.

Test procedures. Of 75 animals given noxious stimulation, $13(17 \%)$ were tested after 7-10 d, $42(56 \%)$ were tested after $11-15 \mathrm{~d}, 14(19 \%)$ were tested after $16-20 \mathrm{~d}$, and $6(8 \%)$ were tested $21-25 \mathrm{~d}$ after stimulation. The proportions in each period were evenly distributed across the groups. Following injection with isotonic $\mathrm{MgCl}_{2}(50-80 \%$ of the animal's weight), each animal was bisected from head to base of the tail, and the viscera and mantle organs were removed. The intact tail, connected to the pedal-pleural ganglia by the posterior pedal nerves, was stretched taut by numerous restraining pins inserted at $1-2 \mathrm{~mm}$ intervals along the entire lateral margin and base of the tail. Right and left pleural ganglia were surgically desheathed to expose bilateral clusters of ventrocaudal (VC) mechanosensory/nociceptive neuron somata (Walters et al., 1983a). Each pleural ganglion and VC cluster was sketched in detail, and a life-sized diagram of the tail and adjacent body surface was made on graph paper. Dividers were used for precise transfer of each measurement from the preparation to the diagram. Tail sensory neurons were impaled with intracellular electrodes (see Walters et al., 1983, for details) about $1 \mathrm{hr}$ after washout of the $\mathrm{MgCl}_{2}$ solution from the tail. Action potentials were displayed on a storage oscilloscope.

Excitatory receptive fields were mapped with a calibrated von Frey hair (Stoelting, Chicago) having a bending pressure of $70 \mathrm{gm} / \mathrm{mm}$. The location of each point eliciting 1 or more action potentials in the soma was measured with dividers from a fixed landmark on the midline at the posterior insertion of the parapodia. The area of each receptive field was measured within the perimeter defined by connecting the outermost excitatory points on the diagram.

Mechanosensory threshold was estimated by applying a series of 10 progressively stiffer calibrated von Frey hairs to the field. These stimuli yielded a discrete scalc of thresholds ranging from $4 \mathrm{gm} / \mathrm{mm}^{2}$ to grcatcr than $75 \mathrm{gm} / \mathrm{mm}^{2}$ (Fig. 3). Each hair was applied briefly (about $0.5 \mathrm{sec}$, see Fig. 2) and repeatedly (5-30 applications per von Frey hair for each cell) at 2-15 sec intervals to different points throughout much of the receptive field (avoiding the margins). Threshold was defined as the lowest pressure that consistently elicited responses from the field. A criterion emphasizing repeatability of responses was used because intermittent "spontaneous" action potentials were occasionally observed (see Results). Specifically, threshold was defined as the lowest bending pressure that elicited 1 or more action potentials in the sensory neuron soma on at least 3 consecutive applications of the von Frey hair to the receptive field. After initial threshold determination, hairs 1 step below and 1 step above the threshold value were tested to verify that action potentials were elicited consistently by stiffer and not by softer hairs. Because the largest possible sample of cells was sought in each preparation and because threshold determination for a single cell could involve over 200 von Frey hair applications, the experimenter did not count the number of action potentials evoked by each stimulus during the experiment. Lack of a tape recorder or chart recorder in most experiments precluded later analysis of possible effects on spike number or pattern.

Three criteria were used to determine if receptive fields crossed the midline. The first was functional overlap with contralateral receptive fields. Functional overlap or crossover was indicated when a stimulus evoked concurrent responses in 2 sensory neurons ( 1 in each pleural ganglion, on opposite sides of the body) innervating adjacent fields on opposite sides of the midline. Additional criteria for extension across the midline were required for fields crossing into denervated regions posterior to the cut. Midway through the study we noticed that a discrete reddish pigment stripe was often present and congruent with the functionally determined midline. In 21 of 40 cut preparations this midlinc stripe was used as a marker for the midline posterior to the cut. A midline pigment stripe has been used similarly as a landmark in receptive field studies in the leech (Blackshaw et al., 1982). In animals in which the pigment stripe was not observed, receptive field crossover posterior to the cut was determined by caudal extrapolation of the midline defined by the functional boundary between contralateral receptive fields anterior to the cut.

Statistical analysis. Nonparametric statistics were used to evaluate differences in threshold, receptive field area (because of positive skew), and crossover frequency. Central tendency was represented by medians, and dispersion by interquartile ranges. If overall effects were established using a Kruskal-Wallis test, planned comparisons were made between the Control group and each of the 3 stimulated groups using 2-tailed Mann-Whitney $U$ tests. Normally distributed data (degrec of receptive field crossover) were analyzed with a 1-way ANOVA and Scheffe's test for multiple comparisons.

\section{Results}

\section{Rapid adaptation of mechanosensory responses to von Frey hair stimulation}

An unexpected but useful observation during the present study was that responses to stimulation with thin $(0.1-$ to $0.6-\mathrm{mm}$ diameter) von Frey hairs adapted rapidly. Figure 1 illustrates typical responses in which the on-response lasted less than 0.3 sec, even when von Frey hair application (in this case driven by a solenoid and timed electronically) was prolonged to $1 \mathrm{sec}$. Also shown is a brief, constant-latency off-response, which was often observed in tail sensory neurons. The rapidly adapting response to punctate stimulation contrasts with the slow adaptation observed to stimuli delivered with a wider $5 \mathrm{~mm}$ probc (Walters et al., 1983). The difference in adaptation might be due to different mechanical effects of the stimuli or to spatial integration by the peripheral sensory arbor.

Examination of strain gauge recordings during 121 hand-held von Frey hair $\left(5-70 \mathrm{gm} / \mathrm{mm}^{2}\right)$ applications showed that stimulus durations ranged from 0.4 to $1.0 \mathrm{sec}$, and averaged $0.5 \mathrm{sec}$. Representative responses to hand-held von Frey hairs are il-. lustrated in Figure 2. In this case, a von Frey hair exerting 25 $\mathrm{gm} / \mathrm{mm}^{2}$ evoked no responses (not shown), a hair exerting 30 $\mathrm{gm} / \mathrm{mm}^{2}$ evoked a response on 2 of 17 applications (a failure is shown in Fig. $2 A$ ), and a hair exerting $35 \mathrm{gm} / \mathrm{mm}^{2}$ evoked 3 consecutive responses (Fig. 2, $B-D$ ), fulfilling our criterion for the threshold of consistent responses. Subsequent application of the $30 \mathrm{gm} / \mathrm{mm}^{2}$ hair did not elicit action potentials. In this and most cells, the threshold responses exhibited multiple action 
Figure I. Rapid adaptation of mechanosensory responses to von Frey hair stimulation. The von Frey hair $(45 \mathrm{gm} /$ $\mathrm{mm}^{2}$ bending pressure) was driven by an electronically timed solenoid. Tension across the tail was recorded at the edge of the cell's receptive field with a strain gauge. $A$, Response to $0.5 \mathrm{sec}$ stimulus. The initial quasi-rectangular increase in tension is produced by pressure from the von Frey hair. The subsequent rise in tension is due to the evoked withdrawal reflex. Note that the reflexive withdrawal does not begin until after stimulus offset. $B$, Response to $1.0 \mathrm{sec}$ stimulus. The change in tension is less than observed in $A$ because the von Frey hair was applied at a point more distant from the strain gauge. Neither stimulus produced sensory neuron on-responses that lasted as long as the stimulus, and both produced offresponses.
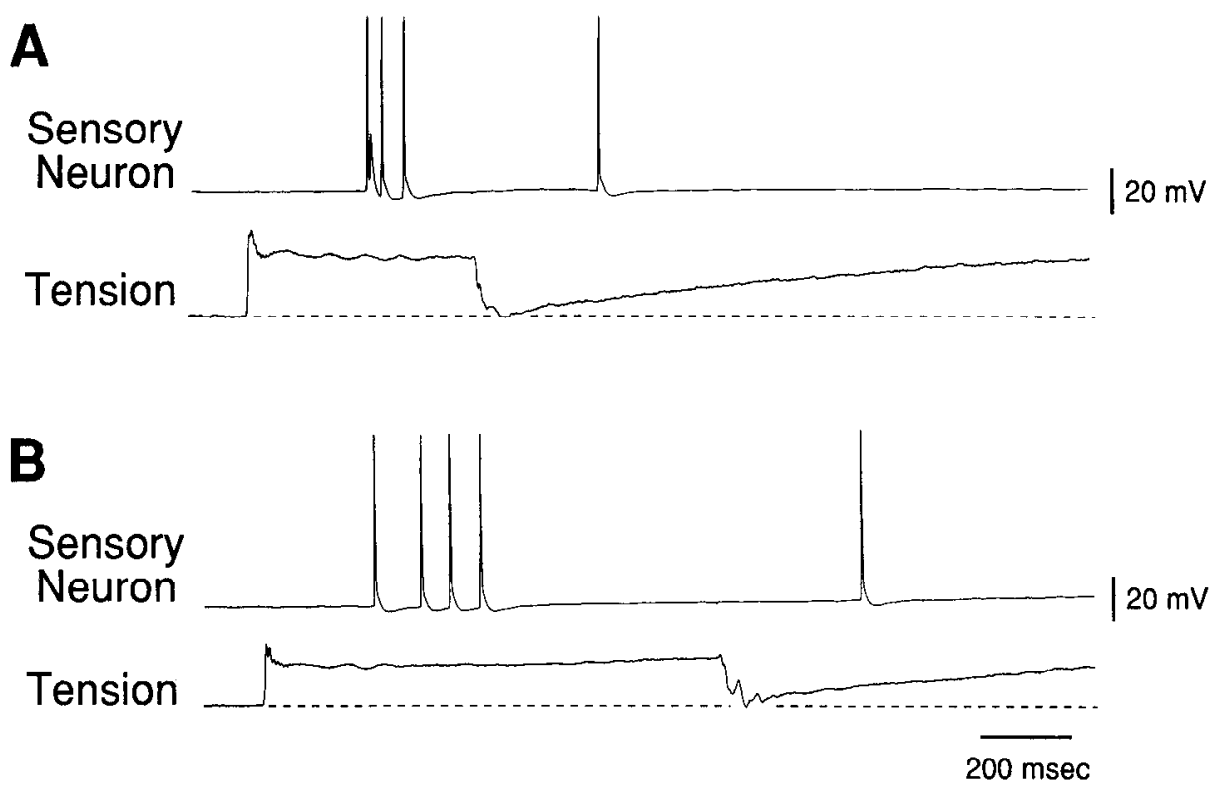

potentials, although in some cells each threshold response was a single action potential.

The rapid adaptation to von Frey hair stimuli lasting about $0.5 \mathrm{sec}$ or longer indicates that the probability of action potential generation and number of action potentials have little if any dependence on stimulus duration. This independence reduces chances that potential unconscious bias could influence responses by variation in the duration of hand-held von Frey hair application (see Discussion).

\section{Long-term reduction of cutaneous response thresholds}

The threshold for consistently eliciting action potentials by test stimulation with von Frey hairs was reduced by noxious stimuli applied to the tail near the cell's receptive field 1-3 weeks earlier in the intact animal (Fig. 3A). Planned comparisons of cells with tail fields adjacent to the midline (proximal to the region of noxious stimulation) showed that cutaneous response thresholds of Cut Alone and Superficial Stimulation cells were significantly lower than the thresholds of Control cells $(p=0.03$ and 0.02 , respectively). Although a trend towards lower thresholds was seen in Cut + Superficial Stimulation cells, thresholds of this group were not significantly different from the Control group. Single action potentials occasionally occurred "spontaneously" in 4 of 16 Control preparations (25\%), 6 of 26 Cut Alone prep- arations $(23 \%), 4$ of 18 Superficial Stimulation preparations $(22 \%)$, and 9 of $31 \mathrm{Cut}+$ Superficial Stimulation preparations (29\%). Usually only 1 to 2 cells (maximum 6) per preparation showed spontaneous activity. No significant differences were found in the incidence of spontaneous activity among the 4 groups. The cause of the spontaneous activity is unknown but may be related to the tail preparation being held taut by numerous pins around its edge.

To test the dependence of threshold changes on proximity to the site of prior noxious stimulation, we examined fields distal to the stimulation site (where they were less likely to have been activated by prior noxious stimulation). Distal fields were defined as fields adjacent to the margin of the foot (except fields contacting the cut) and antcrior ficlds adjacent to the parapodia and midbody region. Distal fields in the 3 stimulated groups showed no significant differences in response threshold compared with fields in the same regions in the unstimulated Control group (Fig. 3B), suggesting that long-term threshold reduction is weak or absent in the fields distant from the site of prior noxious stimulation. Although there was a tendency for proximal fields to have lower thresholds than distal fields in Cut Alone and in Superficial Stimulation groups, direct comparison of the thresholds of distal and proximal fields did not reveal significant differences in any of the groups. A direct compar-

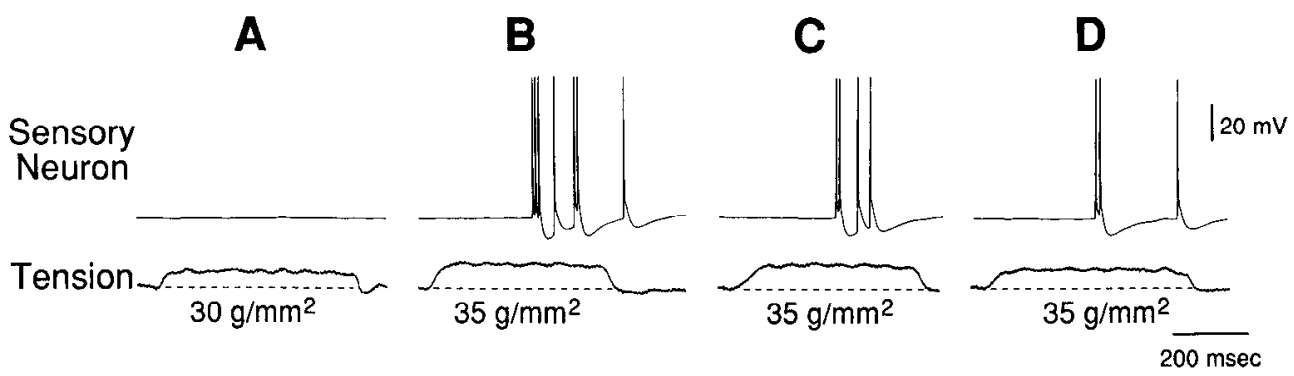

Figure 2. Threshold responses to hand-held von Frey hairs. The bending pressures of the von Frey hairs are indicated beneath the records of tail tension. $A$, Lack of response to subthreshold stimulus. $B-D$, Consecutive responses fulfilling criterion for consistent response threshold. This cell did not exhibit off-responses (record segments not shown). Tension records show that each von Frey hair stimulus lasted about 0.5 sec and was relatively repeatable. The cell is different from that shown in Figure 1, but the animal is the same (trained $18 \mathrm{~d}$ earlier with shock alone). 
ison may be misleading, however, because proximal fields tended to have higher thresholds than distal fields in Control animals. This difference may be related to the fact that body wall tissue in the middle of the tail where the proximal fields were is considerably thicker than the tissue along the margin of the tail where most of the distal fields were. Thicker tissue would be expected to decrease chances of eliciting responses from receptive fields on the ventral side of the tail (which could not be distinguished from dorsal fields by our procedure). In addition, threshold differences may have resulted from distal fields along the tail margin being penetrated by many more restraining pins than were the proximal fields having a border on the midline.

\section{Long-term expansion of receptive fields}

To investigate possible long-term alterations in size and/or shape of excitatory receptive fields following noxious stimulation, each field perimeter was carefully mapped (examples in Fig. 4). The enclosed area was expressed as a fraction of the area of the entire dorsal tail surface (Fig. 5). Planned comparisons of cells with fields proximal to the site of prior noxious stimulation revealed that receptive fields near the midline in the Control group were significantly smaller than corresponding receptive fields in Cut Alone $(p=0.001)$, Superficial Stimulation $(p=0.02)$, or Cut + Superficial Stimulation $(p=0.006)$ groups. In this analysis, we excluded midline receptive fields in Cut Alone and Cut $+\mathrm{Su}-$ perficial Stimulation groups on the same side as the cut to eliminate receptive fields truncated by the cut. These results show that strong electrical or mechanical stimulation can cause a longterm increase in size of receptive fields near the stimulation site.

\section{Long-term alteration of midline receptive field boundaries}

Previous studies of Aplysia (Jahan-Parwar and Fredman, 1978; Walters et al., 1983) suggested that a boundary exists along the animal's midline separating bilaterally organized mechanosensory receptive fields. Figure $4 A$ illustrates the lack of overlap across the midline of receptive fields on opposite sides of a tail in the Control group. Less than $2 \%$ of receptive fields adjacent to the midline ( 2 of 133) in the Control group crossed over the midline, as indicated by functional overlap with contralateral fields (Fig. 6). Interestingly, both crossover fields in the Control group occurred in animals with scars in the vicinity of the crossover, suggesting that crossover may have been caused by a prior, naturally occurring injury. By contrast, in animals previously exposed to noxious stimulation, receptive fields crossed the midline at a 5- to 10-fold higher frequency than in the Control group (Fig. 6, examples in Fig. 4). Chi-square tests showed frequencies of midline crossover were significantly higher in Cut Alone (15 of 110 fields, $p=0.003$ ), Superficial Stimulation (13 of $126, p$ $=0.003)$, and Cut + Superficial Stimulation $(29$ of $144, p<$ $0.001)$ groups than in the Control group. However, even in preparations exhibiting crossover, only a minority of sampled tail fields crossed the midline. In the 3 experimental groups, 24 preparations exhibited single crossover fields, 11 exhibited 2 crossover fields, 3 exhibited 3 crossover fields, and 2 exhibited 4 crossover fields. In the Cut Alone and Cut + Shock groups crossover fields usually occurred posterior to the cut (14 of 19 and 22 of 29 cases, respectively). Thus, most crossover fields extended into the region deprived of direct neural connections to the CNS (Fig. 4). Not all fields crossed from the uncut to the cut side of the tail: 3 of 19 fields in the Cut Alone group and 1 of 29 fields in the Cut + Shock group crossed from the cut to the uncut side (anterior to the cut). In the Superficial Stimulation

\section{A. Proximal fields}

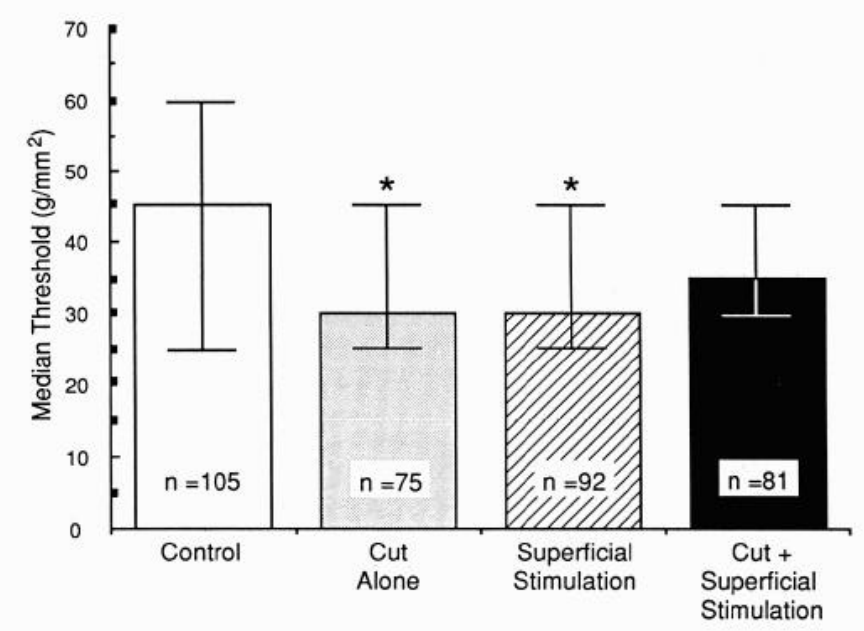

B. Distal fields

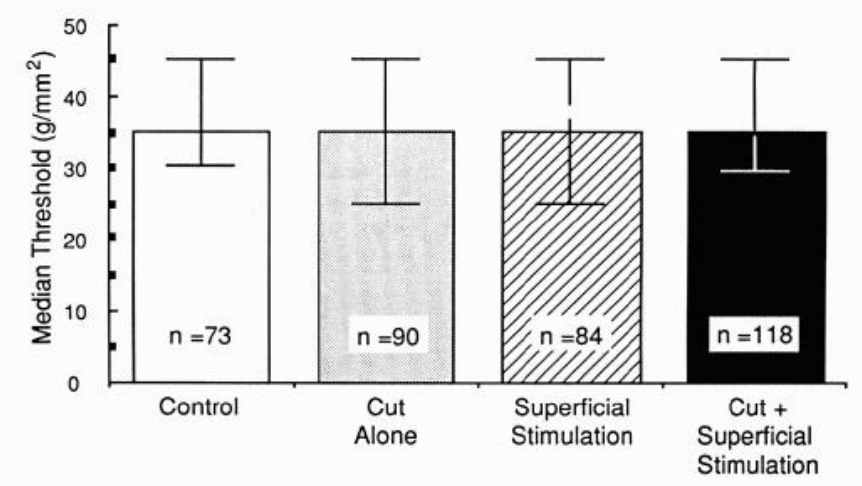

Figure 3. Noxious stimulation causes a long-term decrease in mechanosensory threshold in receptive fields near the site of stimulation but not in distal fields. Thresholds were tested with a series of von Frey hairs of different stiffness. The bending pressure of each hair, rounded to the nearest $5 \mathrm{gm} / \mathrm{mm}^{2}$, is indicated by a filled square on the ordinate. Bending pressures are not shown for the stiffest hairs used in the study. Asterisks in this and subsequent figures indicate significant differences $(p<0.05)$ from the Control group. Error bars show interquartile range. Cells were sampled in 16 Control animals, 26 Cut-Alone animals, 18 Superficial Stimulation animals, and 31 Cut + Superficial Stimulation animals. $A$, Receptive fields proximal to the site of noxious stimulation (fields near the midline of the tail). $B$, Receptive fields distal to the site of noxious stimulation (on the lateral and anterior borders of the tail).

group, 8 fields crossed from left to right and 5 from right to left (Fig. 4C).

The 3 noxious stimulation procedures produced different degrees of receptive field extension across the midline in those fields that showed crossover. This was expressed as a significant overall effect of stimulation procedure on the fraction of each cell's total field area found on the contralateral side of the midline $\left(F_{2,55}=11.87, p<0.001\right)$. Multiple comparisons showed that the contralateral fraction of each crossover field was significantly larger in the Superficial Stimulation group than in the Cut Alone or Cut + Superficial Stimulation groups $(p<0.005$ in each case). Thus, although the Superficial Stimulation protocol produced the lowest frequency of receptive field crossover, the crossover fields in this group exhibited the largest degree of crossover per field. Indeed, in 4 of 13 crossover fields in the Superficial Stimulation group (versus 2 of 19 fields in the Cut Alone group and 0 of 29 fields in the Cut + Stimulation group), 

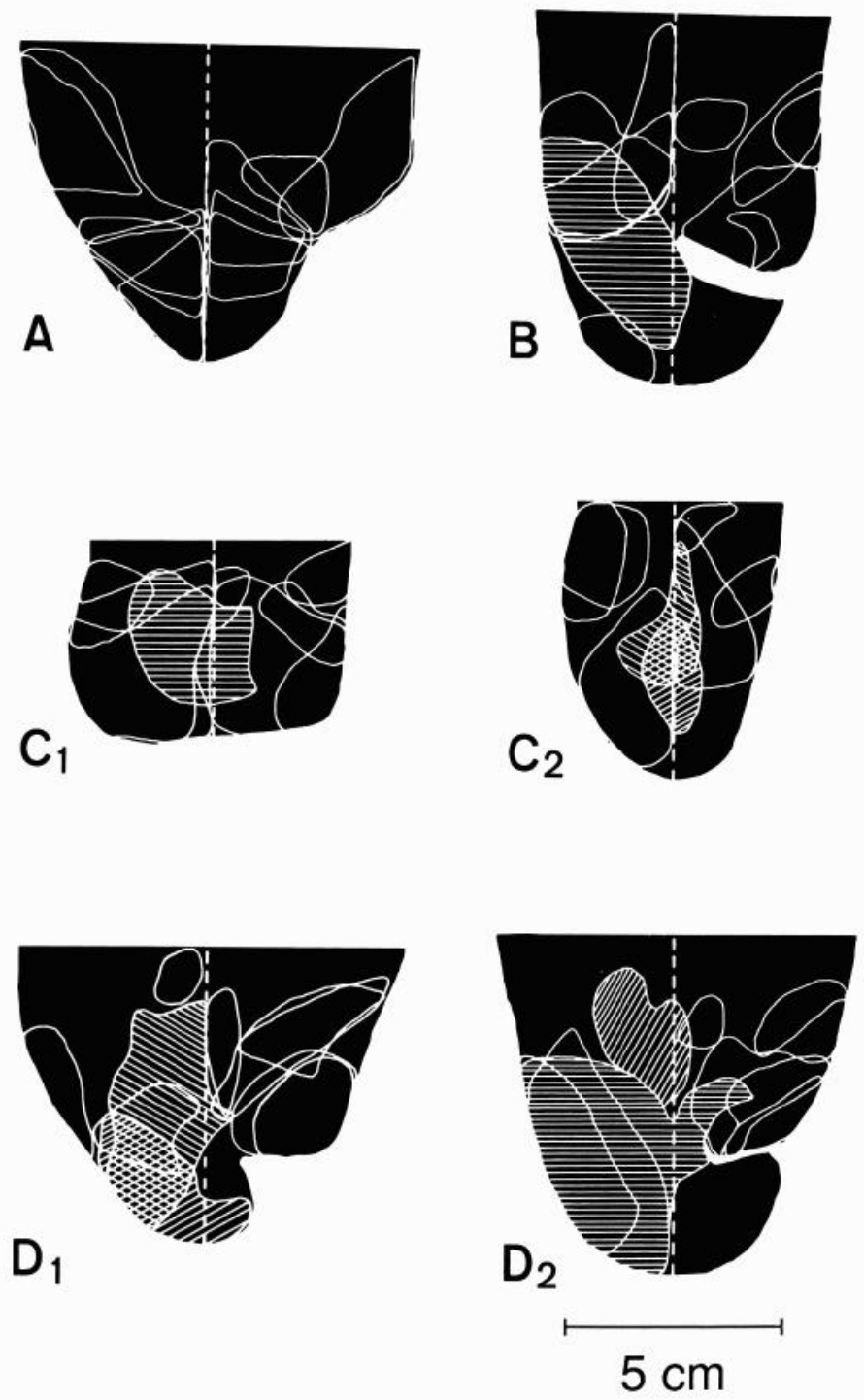

Figure 4. Examples of mechanosensory receptive fields on normal tail and tails previously given noxious stimulation. Individual excitatory fields are circumscribed by continuous lines. The dashed line indicates the midline as determined by the functional boundary between contralateral fields and by a pigmented midline stripe. Receptive fields that cross the midline are indicated with hatching. $A$, Control tail. No fields cross the midline or overlap with contralateral fields. $B$, Cut Alone. One field on the left shows a small degree of crossover posterior to the cut on the right. $C$, Superficial Stimulation. $C 1$, A single field crosses from the left to the right. $C 2$, Two fields cross from the right to the left. $D$, Cut + Stimulation. D1, One field crosses posterior to the cut and 1 crosses anterior to the cut. D2, One field crosses anterior to the cut and 1 field crosses anterior and posterior to the cut.

the contralateral part of the receptive field was actually larger than the ipsilateral part of the field.

To determine whether long-term changes in size and shape of receptive fields produced by noxious stimulation depended upon peripheral synaptic input to the sensory neuron during testing, we examined the effects of injecting artificial seawater containing 10 or $30 \mathrm{~mm} \mathrm{CoCl}_{2}$ into receptive fields of animals in the Cut + Superficial Stimulation group. These solutions block $\mathrm{Ca}^{2+}$ conductances and central synaptic transmission in Aplysia (Byrne et al., 1979; Walters et al., 1983). When injected into the sinus between the skin and muscle layer these solutions

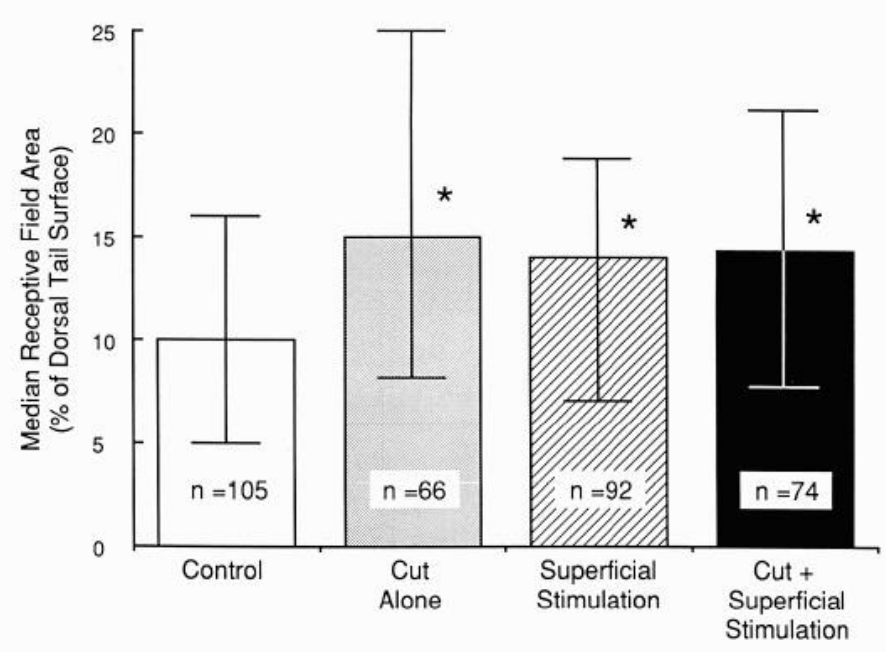

Figure 5. Noxious stimulation causes a long-term increase in mechanosensory receptive field area. Areas are expressed as a percentage of the dorsal surface area of the whole tail. Error bars show interquartile ranges.

block body wall contractions evoked by stimulation of skin or nerves within a few minutes (Krontiris-Litowitz et al., 1989; A. L. Clatworthy and E. T. Walters, unpublished observations). $\mathrm{CoCl}_{2}$ injection did not change the borders of either noncrossover $(n=10)$ or crossover fields $(n=4)$ in the Cut + Superficial Stimulation group when tested $10-20 \mathrm{~min}$ after injection.

\section{Discussion}

Modulatory signals likely to be released by noxious stimulation of Aplysia produce a range of cellular alterations linked to enhancement of synaptic transmission and soma excitability in mechanosensory neurons (reviewed by Kandel and Schwartz, 1982; Byrne, 1987). The demonstration that effects of extrinsic modulators can be amplified by sensory neuron activation initially led to the hypothesis that activity-dependent extrinsic modulation (ADEM) is a mechanism of associative learning (Hawkins et al., 1983; Walters and Byrne, 1983). The more inclusive term "ADEM" (Walters, 1987a) is used by the present authors rather than "activity-dependent presynaptic facilitation" (Hawkins et al., 1983) or "activity-dependent neuromodulation" (Walters and Byrne, 1983) to allow for nonsynaptic loci and non-neuronal mediators. Walters (1987a, b) argued that ADEM of Aplysia mechanosensory neurons is produced most effectively under natural conditions by stimuli that injure the sensory neuron's receptive field. Furthermore, it was shown that trauma-induced ADEM can produce a site-specific, central sensory memory of body-wall trauma functionally similar to longterm primary hyperalgesia in mammals (cf. Woolf, 1984; Walters, $1987 \mathrm{a}, \mathrm{b}$ ). The present study was designed to investigate a corollary of this view-if the function of the observed central sensory effects is to enhance sensory signals from an injured region, then this same function might also be served by boosting peripheral sensitivity of sensory cells innervating the injured region.

We found several changes in peripheral receptive fields $1-3$ weeks after noxious tail stimulation. Tail mechanosensory neuron thresholds were reduced, tail receptive fields were enlarged, and the probability of functional expansion across the tail midline was increased. These effects were produced by cutting or shocking the tail, or by combining a cut with shock or pinch. 
The effects from combining a cut with superficial stimulation were generally no stronger than the effects of either stimulus alone, and indeed may have been weaker on threshold (Fig. 3A). Although the slight difference in threshold between the Cut + Superficial Stimulation group and the other stimulated groups may simply reflect statistical variation, it is also possible that these 2 forms of stimulation interfere with each other in some way (e.g., contraction evoked by the cut may alter the effectiveness of shock application, or the shock may disturb recuperative responses to the cut). Electric shock-a strong, unnatural stimulus - probably produces complex and abnormal peripheral effects in addition to its potent activation of mechanosensory neurons.

Although a completely blind procedure was not feasible, it is unlikely that unconscious tester bias can account for our observations. First, testing was conducted with flexible von Frey hairs, commonly used in somatosensory studies (e.g., Diamond, 1982; Thalhammer and LaMotte, 1982) because bending of the hair absorbs additional force applied above the bending point. In addition, rapid adaptation of the sensory response during von Frey hair application made it unlikely that differences in stimulus duration could affect the probability of a response. Indeed, in separate experiments we have been unable to produce artificial differences in threshold by deliberately increasing the force applied to a given von Frey hair. Second, our index of threshold and receptive field geometry - the consistent presence of all-or-none action potentials-left little room for subjective interpretation. Third, the magnitude of stimulation-induced crossover effects compared with the control pattern argues against the possibility that these effects are merely due to unconscious bias during testing. Crossover occurred in only 2 of 133 midline fields in the Control group, while 57 of 380 fields crossed the midline in the 3 stimulated groups. Our indices of receptive field crossover were sensitive enough to detect small degrees of crossover ( $<2 \%$ of a field's area as indicated by simultaneous recordings from contralateral neighbors), but many examples of crossover in the stimulated groups were quite large. For example, in 6 of 57 crossover fields detected in the experimental groups (and none in the Control group), the extended, contralateral portion of the receptive field was larger than the ipsilateral portion of the field. Receptive field boundaries were remarkably stable during each test session, and both control and crossover fields showed no change in geometry when perfused with cobalt solutions.

\section{Loci underlying long-term receptive field plasticity}

The long-term changes observed in mechanosensory receptive fields might be due to (1) alterations in cells presynaptic to the recorded mechanosensory neurons, (2) an increase in responsiveness of preexisting receptive processes of the recorded mechanosensory neurons, (3) sprouting of new receptive processes from mechanosensory neurons, and/or (4) alterations in the probability of action potential conduction in peripheral or central components of the mechanosensory neurons. Although we cannot exclude a contribution from cells presynaptic to the $\mathrm{VC}$ mechanosensory neurons, this locus is unlikely to be of major importance since VC cells appear to be primary sensory neurons (Walters et al., 1983) and cobalt concentrations that are likely to have blocked or largely reduced chemical synaptic transmission in the tail had no effect on receptive field geometry in stimulated animals. We conclude, therefore, that the long-term alterations are probably due to enhanced responsiveness of the

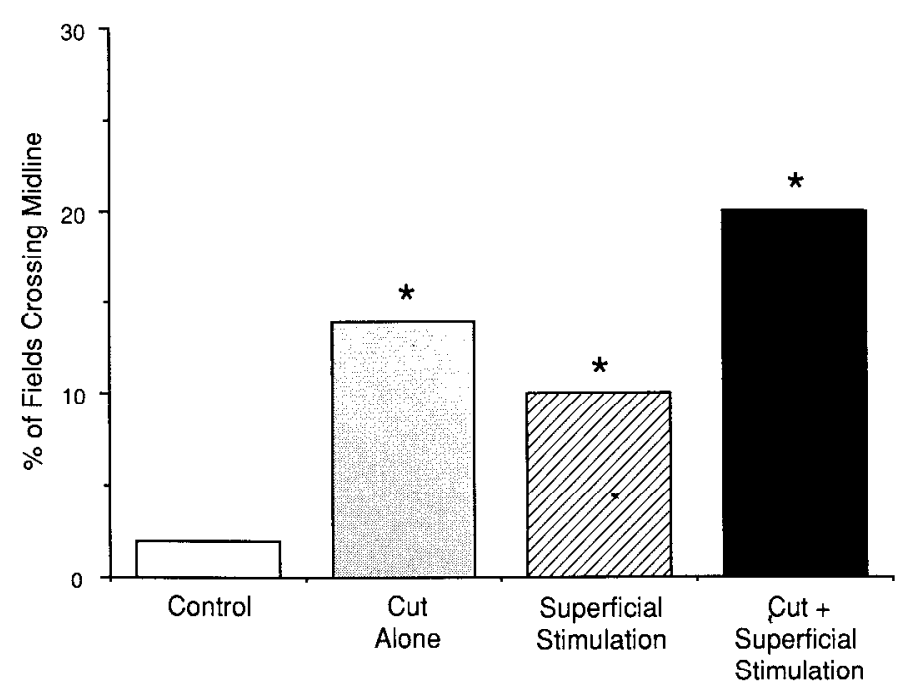

Figure 6. Noxious stimulation causes a long-term increase in frequency of receptive field crossover across the tail midline. The frequency of receptive fields crossing the midline is expressed as a percentage of all cells found with tail receptive fields adjacent to the midline in each preparation.

VC mechanosensory neurons. This enhanced responsiveness might reflect changes in the mechanical properties of the body wall rather than the sensory neuron itself, but this possibility seems unlikely since the lowest thresholds within each field showed no tendency to cluster near the cut or regions of shockinduced fading, where the mechanical changes should have been greatest. In addition, mechanical alterations do not account in a straightforward way for the increase in receptive field size. Swelling of the tail (which if present was not noticeable) cannot explain the changes in area since the field areas were normalized to the total tail area.

While it is likely that receptive field changes reflect alterations intrinsic to the sensory neurons, we do not yet know the extent to which such changes are due to changes in the excitability of preexisting processes or sprouting of new processes. Technical obstacles to selective introduction of dye into peripheral processes of VC sensory neurons have precluded direct morphological analysis that might demonstrate conclusively whether noxious stimulation induces peripheral sprouting. Indirect evidence, however, supports this possibility. We have recently found that peripheral excitability increases acutely after perfusion of 5 -HT into receptive fields of undamaged animals, without changing receptive field geometry (Billy and Walters, 1988). Increased excitability without an increase in receptive field size suggests, but does not prove, that the VC sensory neurons in this preparation lack "silent," subthreshold fringes around their receptive fields, which would be functionally unmasked by increases in excitability (cf. Wall, 1977). Some primary mechanoreceptors in vertebrates also appear to lack subthreshold fringes (Diamond, 1982). If subthreshold fringes arc absent, long-term increases in receptive field size would not be a consequence of increased excitability of preexisting fibers but would, instead, require peripheral growth. Peripheral sprouting of mechanosensory neurons in Aplysia is plausible since peripheral sensory sprouting has been described in animals ranging from invertebrates to humans (e.g., Blackshaw et al., 1982; Diamond, 1982; Inbal et al., 1987), and many molluscan neurons sprout readily in response to damage or stress (Bulloch, 1985). Moreover, re- 
Figure 7. Whole-cell model of trauma-induced mechanosensory plasticity. $A$. Immediate response to peripheral trauma. 1, A traumatic stimulus injures and strongly activates peripheral receptive processes of the mechanosensory/ nociceptive neuron. 2 , The resulting action potentials propagate to the synapses and soma. 3, This and other activated sensory neurons trigger the release of chemical modulators from a central and peripheral facilitatory network. These extrinsic chemical signals feed back on to the activated sensory neuron, causing facilitatory effects at the soma, synapses, and peripheral receptive processes that are amplified by the preceding spike activity (ADEM). 4, Amplified levels of modulator-induced intracellular messengers in the soma stimulate the synthesis and export of growth-associated proteins for central and peripheral structural changes. $B$, Intermediate-term memory of trauma is expressed as enhanced cellular signaling strength caused by increased excitability of peripheral and central processes (indicated by thickened lines) and enhanced transmitter release. These effects probably involve decreased $\mathrm{K}$ conductances. $C$, Long-term increases in signaling effectiveness are produced by growth of both the central synaptic arbor and peripheral receptive processes. Peripheral growth is expressed as regenerative and collateral sprouting. The excitability of preexisting processes may also remain elevated.
A.

Noxious Stimulation

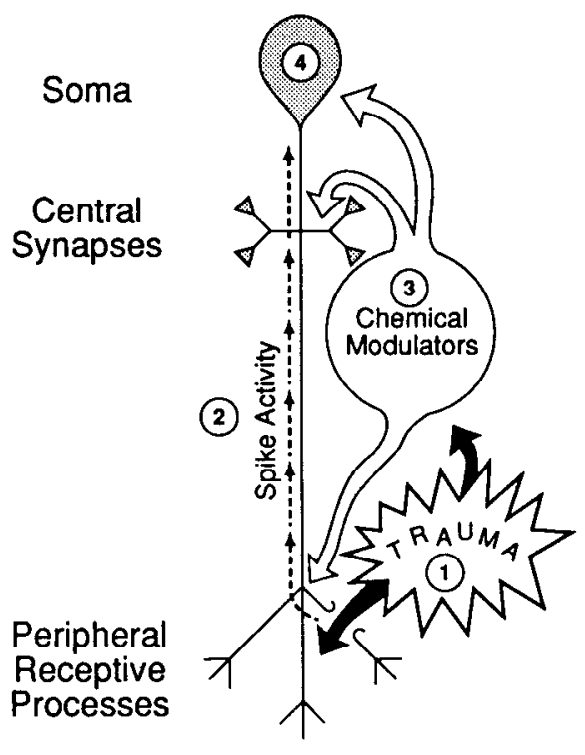

Activity-dependent extrinsic modulation (ADEM)
B. Hours-Days Afterwards

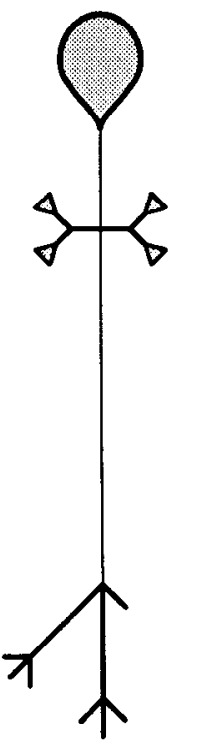

$$
\begin{gathered}
\uparrow \text { Excitability }\left(\downarrow g_{K}\right) \\
\uparrow \underset{\text { release }}{\text { Transmitter }}
\end{gathered}
$$

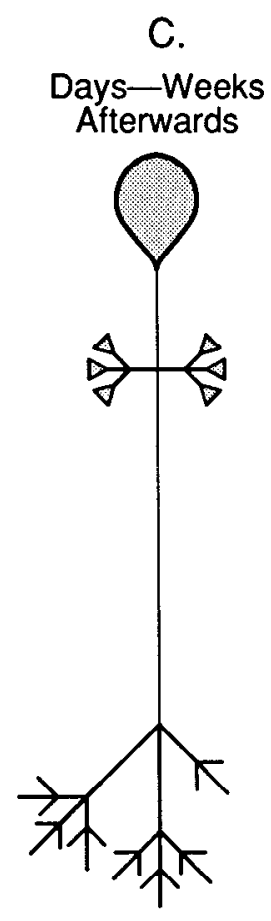

Central and peripheral sprouting peated, intense noxious stimulation is reported to induce central structural changes in mechanosensory neurons of Aplysia, including growth of the cell's central arbor (Bailey and Chen, 1983, 1988).

An interesting possibility is that receptive field plasticity involves mechanisms downstream from the transduction process that increase the probability that action potentials generated by weak stimuli or in fringes of the receptive field will be conducted out of the tail and/or into the central soma and synapses. Supporting this general possibility, intense nerve stimulation can produce short-term changes in central conduction properties within the isolated CNS (Clatworthy and Walters, 1988). While central conduction changes might affect threshold measurements, it is difficult for central conduction changes to explain alterations in receptive field geometry since most tail sensory neurons have only a single axon entering the CNS (Cleary and Byrne, 1984). More plausible is that changes in peripheral conduction contribute to alterations in receptive field geometry, alterations that might involve mechanisms shared with longterm excitability changes in the soma (Dale et al., 1987; Scholz and Byrne, 1987; Walters, 1987b). It should be emphasized that, whether the receptive field alterations we describe are due to peripheral or central sensory changes, or to both, the effective receptive field (as represented in the CNS) is sensitized and enlarged for several weeks following nearby noxious stimulation. The preceding arguments suggest that the effective receptive field may be enhanced by both pcripheral and central mechanisms, supporting the following model.

\section{Activity-dependent model of whole-cell sensory plasticity}

The results of this study, combined with previous descriptions of central effects of noxious stimulation on mechanosensory neurons in Aplysia, suggest that long-term, mechanosensory plasticity involves an increase in signaling strength of the entire sensory cell (Fig. 7). Because body wall injury under normal conditions causes activation of sensory neurons with damaged fields and release of chemical modulators (Walters, 1987a, b; Krontiris-Litowitz et al., 1989), and because ADEM has been implicated in long-term sensory enhancement following injury (Walters, 1987a, b), we propose that injury-induced ADEM boosts the long-term signaling strength of the whole cell, including its peripheral receptive field. Although we have not directly demonstrated a role for activity, extrinsic modulation, or ADEM in the periphery, this hypothesis is consistent with out present results and with other physiological parallels between central and peripheral regions of the sensory neurons (Clark and Kandel, 1984; Klein et al., 1986; Billy and Walters, 1988). By this hypothesis, mechanical trauma or electric shock of the body surface activates mechanosensory/nociceptive neurons, in turn activating facilitatory interneurons in the CNS (Hawkins et al., 1981; Mackey et al., 1986) and PNS. These cells release chemical modulators onto the sensory neurons, enhancing excitability and transmitter release centrally and peripherally. Sensory neurons activated by trauma undergo activity-dependent amplification of the ccllular effects of the modulators (ADEM), increasing and prolonging the cellular 
sensitization relative to the modulator-induced changes in unactivated sensory neurons having fields outside the region of trauma (site-specific sensitization). A parsimonious mechanism that might contribute to both the central and peripheral signaling effects is activity-dependent (Hawkins et al., 1983; Walters and Byrne, 1983; Abrams and Kandel, 1988) enhancement of the 5 -HT-induced reduction in potassium conductances previously described in the sensory cell soma (Klein and Kandel, 1980; Klein et al., 1982; Walsh and Byrne, 1984; Pollock et al., 1985).

The expansion of receptive fields observed 1-3 weeks after noxious stimulation, coupled with the known capacity of mechanosensory neurons in adult Aplysia for sensitization-related growth within the CNS (Bailey and Chen, 1983, 1988) suggests that injury may induce morphological growth in traumatized receptive fields. This postulate has interesting functional implications. Regenerative sprouting of receptive arbors of injured sensory neurons and collateral sprouting of surrounding sensory neurons into a damaged region should compensate at least partially for loss of sensory function. Growth of nearby receptive arbors might increase the density (overlap) of receptive fields around an injury and boost reflex sensitivity by increasing the number of sensory neurons that will fire to a stimulus near the injury.

Trauma-induced growth of peripheral sensory processes is likely to depend upon various peripheral and central influences. Peripheral influences may be complex, possibly involving alteration of competitive interactions among fields (e.g., Diamond, 1982; Murphey, 1985) and local levels of growth factors (e.g., Thoenen and Edgar, 1985). The fact that only a minority of receptive fields extended across the midline after noxious stimulation indicates that there are strong peripheral constraints on receptive field growth in this system and that sprouting may normally be limited to a restricted domain (Diamond, 1982). Indeed, extension across the midline may depend upon at least 2 effects of trauma: induction of receptive field growth and perturbation of spatial cues that define the midline.

A likely central contribution to peripheral sprouting is the provision of proteins for growth and repair. The dependence of growth upon protein synthesis suggests an interesting potential function for ADEM in the soma. Because the soma is off the direct line of communication between the cell's peripheral receptive processes and its synaptic terminals, it seems unlikely that ADEM of the soma has important immediate effects on transmission of sensory information. The soma is, however, probably where growth-associated protcins are synthesized (e.g., Skene and Willard, 1981; Schwartz, 1985). Because the central somata of tail sensory neurons in Aplysia are often $10-15 \mathrm{~cm}$ away from their peripheral receptive fields, a signal for injuryinduced protein synthesis that depended on retrograde axonal transport could take days to reach the soma. In contrast, traumaevoked signals producing ADEM of the soma arrive from the periphery within a fraction of a second. These signals will be a good indicator of injury since ADEM occurs preferentially in sensory neurons that are strongly activated by trauma (Walters, $1987 \mathrm{~b})$. Supporting this hypothesis, both an extrinsic modulator (5-HT) and an intracellular messenger (cyclic AMP) which appear to be involved in ADEM of the soma (Kandel et al., 1983; Ocorr et al., 1985) have been implicated as inducers of protein synthesis in Aplysia (Montarolo et al., 1986; Eskin et al., 1988). The potential advantages of ADEM of the soma for selectively and rapidly triggcring cxport of growth-associated proteins in response to injury of distant processes might have wide signif- icance. For example, noxious cutaneous and nerve stimulation in the rat dramatically accelerate peripheral sprouting of nociceptors into denervated skin - but only if activity is allowed to reach central regions of the nociceptors where the somata are (Nixon et al., 1984; Doucette and Diamond, 1987). Although this acceleration might be due to activity alone, the likelihood that noxious cutaneous and nerve stimulation release heterosynaptic or humoral signals suggests that injury-related ADEM of soma functions may be involved.

Activity-dependent mechanisms are not necessary for longterm sensory alterations in Aplysia since repeated experience (16-20 trials at approximately hourly or daily intervals) with severe shock eventually lcads via extrinsic modulatory pathways to changes in unactivated sensory neurons (Bailey and Chen, 1983, 1988; Frost et al., 1985), including increased synthesis of some proteins (Castellucci et al., 1987). However, the intense stimuli used in long-term sensitization studies of Aplysia evoke prolonged defensive responses like those produced by body wall injury and thus either cause injury or mimic the afferent information associated with body wall injury (Walters and Erickson, 1986; Walters, 1987a, b). Under natural conditions, gradual induction of long-term sensitization by repeated injury (extrinsic modulation alone) is probably very rare, since each additional injury decreases chances that the animal will survive. By contrast, ADEM can induce long-term changes in activated sensory neurons after a single $45 \mathrm{sec}$ exposure to an injurious stimulus (Walters, 1987a, b). In principle, sensory activation alone (without extrinsic modulation) might also be a signal for injury and long-term sensitization. Activation alone is not, however, a good candidate for triggering persistent whole-cell changes since intracellular activation alone causes no long-lasting synaptic facilitation when examined for several hours after activation (Walters and Byrne, 1985), and weak cutaneous stimuli that produce sensory activation but no sign of extrinsic modulation do not lead to long-term, site-specific sensitization (Walters, 1987a). These observations suggest that ADEM is important for the rapid induction of long-term sensitization around wounds and that it may be a signal for trauma-induced changes in receptive field geometry.

\section{References}

Abrams, T. W., and E. R. Kandel (1988) Is contiguity detection in classical conditioning a system or a cellular property? Learning in Aplysia suggests a possible molecular site. Trends Neurosci. 11: 128135.

Bailey, C. H., and M. Chen (1983) Morphological basis of long-term habituation and sensitization in Aplysia. Science 220:91-93.

Bailcy, C. H., and M. Chen (1988) Long-term memory in Aplysia modulates the total number of varicosities of single identified sensory neurons. Proc. Natl. Acad. Sci. USA 85: 2373-2377.

Billy, A. J., and E. T. Walters (1987) Receptive field plasticity and somatotopic organization of pleural mechanosensory/nociceptive neurons of Aplysia. Soc. Neurosci. Abstr. 13: 1393.

Billy, A. J., and E. T. Walters (1988) Sensitization and sensory signals in Aplysia I. Neuromodulator effects on peripheral excitability. Soc. Neurosci. Abstr. 14: 608.

Blackshaw, S. E., J. G. Nicholls, and I. Parnas (1982) Expanded receptive fields of cutaneous mechanoreceptor cells after single neuron deletion in leech central nervous system. J. Physiol. (Lond.) 326: 261268.

Bulloch, A. G. M. (1985) Development and plasticity of the molluscan nervous system. In The Mollusca, Vol. 8: Neurobiology and Behavior, Part 1, A. O. D. Willows, ed., pp. 335-410, Academic, New York.

Byrne, J. H. (1987) Cellular analysis of associative learning. Physiol. Rev. 67: 329-439.

Byrne, J. H., E. Shapiro, N. Dieringer, and J. Koester (1979) Bio- 
physical mechanisms contributing to inking behavior in Aplysia. J. Neurophysiol. 42: 1233-1250.

Castellucci, V. F., E. R. Kandel, T. E. Kennedy, and P. Goelet (1987) Learning associated proteins (LAPs) following long-term sensitization of the gill and siphon withdrawal reflex in Aplysia. Soc. Neurosci. Abstr. 13: 390.

Clark, G. A., and E. R. Kandel (1984) Branch-specific heterosynaptic facilitation in Aplysia siphon sensory cells. Proc. Natl. Acad. Sci. USA 81: 2577-2581

Clark, G. A., and E. R. Kandel (1987) Serotonin produces long-term facilitation at peripheral synapses when applied selectively to synaptic regions of Aplysia siphon sensory cells. Soc. Neurosci. Abstr. 13: 390.

Clatworthy, A. L., and E. T. Walters (1988) Sensitization and sensory signals in Aplysia II. Modulation of central bursting and spike conduction. Soc. Neurosci. Abstr. 14: 608.

Cleary, L. J., and J. H. Byrne (1984) Light and electron microscopic examination of sensory neurons and motoneurons mediating the tail withdrawal reflex in Aplysia. Soc. Neurosci. Abstr. 10: 916.

Coderre, T. J., and R. Melzack (1987) Cutaneous hyperalgesia: Contributions of the peripheral and central nervous systems to the increase in pain sensitivity after injury. Brain Res. 404: 95-106.

Dale, N., E. R. Kandel, and S. Schacher (1987) Serotonin produces long-term changes in the excitability of Aplysia sensory neurons in culture that depend on new protein synthesis. J. Neurosci. 7: 22322238 .

Dale, N., S. Schacher, and E. R. Kandel (1988) Long-term facilitation in Aplysia involves increase in transmitter release. Science 239: 282285.

Diamond, J. (1982) Modeling and competition in the nervous system: Clues from the sensory innervation of skin. Curr. Top. Dev. Biol. 17: 147-205.

Dickhaus, H., M. Zimmermann, and Y. Zotterman (1976) The development in regenerating cutaneous nerves of $\mathrm{C}$ fibre receptors responding to noxious heating of the skin. In Sensory Function of the Skin in Primates, Y. Zotterman, ed., pp. 415-425, Pergamon, Oxford, UK.

Doucette, R., and J. Diamond (1987) Normal and precocious sprouting of heat nociceptors in the skin of adult rats. J. Comp. Neurol. 261: 592-603.

Eskin, A., J. H. Byrne, and K. S. Garcia (1988) Identification of proteins that may mediate sensitization in Aplysia. Soc. Neurosci. Abstr. 14: 838 .

Fitzgerald, M. (1979) The spread of sensitization of polymodal nociceptors in the rabbit from nearby injury and by antidromic nerve stimulation. J. Physiol. (Iond.) 297: 207-216.

Frost, W. N., V. F. Castellucci, R. D. Hawkins, and E. R. Kandel (1985) Monosynaptic connections made by the sensory neurons of the gilland siphon-withdrawal reflex in Aplysia participate in the storage of long-term memory for sensitization. Proc. Natl. Acad. Sci. USA 82 $8266-8269$

Hawkins, R. D., V. F. Castellucci, and E. R. Kandel (1981) Interneurons involved in mediation and modulation of gill-withdrawal reflex in Aplysia. II. Identified neurons produce heterosynaptic facilitation contributing to behavioral sensitization. J. Neurophysiol. 45: 315326.

Hawkins, R. D., T. W. Abrams, T. J. Carew, and E. R. Kandel (1983) A cellular mechanism of classical conditioning in Aplysia: Activitydependent amplification of presynaptic facilitation. Science 219:400404.

Inbal, R., M. Rousso, H. Ashur, P. D. Wall, and M. Devor (1987) Collateral sprouting in skin and sensory recovery after nerve injury in man. Pain 28: 141-154.

Jahan-Parwar, B., and S. M. Fredman (1978) Pedal locomotion in Aplysia californica. I. Sensory and motor fields of pedal nerves. Comp. Biochem. Physiol. 60A: 459-465.

Kandel, E. R., and J. H. Schwartz (1982) Molecular biology of learning: Modulation of transmitter release. Science 218: 433-444.

Kandel, E. R., T. M. Abrams, L. Bernier, T. J. Carew, R. D. Hawkins, and J. H. Schwartz (1983) Classical conditioning and sensitization sharc aspects of the same molccular cascade in Aplysia. Cold Spring Harbor Symp. Quant. Biol. 48: 821-830.

Klein, M., and E. R. Kandel (1980) Mechanism of calcium current modulation underlying presynaptic facilitation and behavioral sensitization in Aplysia. Proc. Natl. Acad. Sci. USA 77: 6912-6916.

Klein, M., J. Camardo, and E. R. Kandel (1982) Serotonin modulates a specific potassium current in the sensory neurons that show presynaptic facilitation in Aplysia. Proc. Natl. Acad. Sci. USA 79:57135717.

Klein, M., B. Hochner, and E. R. Kandel (1986) Facilitatory transmitters and cAMP can modulate accommodation as well as transmitter release in Aplysia sensory neurons: Evidence for parallel processing in a single cell. Proc. Natl. Acad. Sci. USA 83: 7994-7998.

Krontiris-Litowitz, J. K., B. F. Cooper, and E. T. Walters (1989) Humoral factors released during trauma of Aplysia body wall. I. Body wall contraction, cardiac modulation, and central reflex suppression. J. Comp. Physiol. B. (in press).

Lewis T. (1942) Pain, Macmillan, New York.

Mackey, S. L., R. D. Hawkins, and E. R. Kandel (1986) Neurons in 5-HT containing region of cerebral ganglia produce facilitation of LE cells in Aplysia. Soc. Neurosci. Abstr. 12: 1340.

Montarolo, P. G., P. Goelet, V. F. Castellucci, J. Morgan, E. R. Kandel, and S. Schacher (1986) A critical period for macromolecular synthesis in long-term heterosynaptic facilitation in Aplysia. Science 234: 1249-1254.

Murphey, R. K. (1985) Competition and chemoaffinity in insect sensory systems. Trends Neurosci. $8: 120-125$.

Nixon, B. J., R. Doucette, P. C. Jackson, and J. Diamond (1984) Impulse activity evokes precocious sprouting of nociceptive nerves into denervated skin. Somatosensory Res. 2: 97-126.

Ocorr, K. A., E. T. Walters, and J. H. Byrne (1985) Associative conditioning analog selectively increases cAMP levels of tail sensory neurons in Aplysia. Proc. Natl. Acad. Sci. USA 82: 2548-2552.

Pinsker, H. M., W. A. Hening, T. J. Carew, and E. R. Kandel (1973) Long-term sensitization of a defensive withdrawal reflex in Aplysia. Science 182: 1039-1042.

Pollock, J. D., L. Bernier, and J. S. Camardo (1985) Serotonin and cyclic adenosine $3^{\prime}: 5^{\prime}$-monophosphate modulate the $S$ potassium current in tail sensory neurons in the pleural ganglion of Aplysia. J. Neurosci. 5: 1862-1871.

Scholz, K. P., and J. H. Byrne (1987) Long-term sensitization in Aplysia: Biophysical correlates in tail sensory neurons. Science $235: 685-$ 687.

Schwartz, J. H. (1985) Synthesis and distribution of neuronal protein. In Principles of Neural Science, E. R. Kandel and J. H. Schwartz, eds., pp. 37-48, Elsevier, New York.

Skene, J. H. P., and M. Willard (1981) Axonally transported proteins associated with axon growth in rabbit central and peripheral nervous systems. J. Cell. Biol. 89: 96-103.

Thalhammer, J. G., and R. H. LaMotte (1982) Spatial properties of nociceptor sensitization following heat injury of the skin. Brain Res. 231: 257-265.

Thoenen, H., and D. Edgar (1985) Neurotrophic factors. Science 229: 238-242.

Wall, P. D. (1977) The presence of ineffective synapses and the circumstances which unmask them. Phil. Trans. R. Soc. London Ser. B 278: $361-372$

Walsh, J. P., and J. H. Byrne (1984) Forskolin mimics and blocks a serotonin-sensitive decreased $\mathrm{K}^{+}$conductance in tail sensory neurons of Aplysia. Neurosci. Lett. 52: 7-11.

Walters, E. T. (1987a) Site-specific sensitization of defensive reflexes in Aplysia: A simple model of long-term hyperalgesia. J. Neurosci. 7: 400-407.

Walters, E. T. (1987b) Multiple sensory neuronal correlates of sitespecific sensitization in Aplysia. J. Neurosci. 7: 408-417.

Walters, E. T., and J. H. Byrne (1983) Associative conditioning of single sensory neurons suggests a cellular mechanism for learning. Science 219: 405-408.

Walters, E. T., and J. H. Byrne (1985) Long-term enhancement produced by activity-dependent modulation of Aplysia sensory neurons. J. Neurosci. 5: 662-672.

Walters, E. T., and M. T. Erickson (1986) Directional control and the functional organization of defensive responses in Aplysia. J. Comp. Physiol. A. 159: 339-351.

Walters, E. T., J. H. Byrne, T. J. Carew, and E. R. Kandel (1983) Mechanoafferent neurons innervating tail of Aplysia. I. Response properties and synaptic connections. J. Neurophysiol. 50: 1522-1542.

Woolf, C. J. (1984) Long-term alterations in the excitability of the flexion reflex produced by peripheral tissue injury in the chronic decerebrate rat. Pain $18: 325-343$. 